Pacific Journal of Mathematic 


\section{FINITISTIC GLOBAL DIMENSION FOR RINGS}

\section{HoRACE MochizUKI}

The finitistic global dimensions $\operatorname{lf} P D(R), l F P D(R)$, and $l F I D(R)$ are defined for a ring $R$. We obtain the following results for $R$ semiprimary with Jacobson radical $N . C$ is a simple left $R$-module and $l \cdot \operatorname{dim}_{R} C<\infty$, and suppose that $l . \operatorname{dim}_{R} N^{i-1} / N^{i}<\infty$ for $i \geqq 3$. Then $m \leqq l f P D(R)=$ $l F P D(R) \leqq(m+1)$. Theorem 2: Suppose that $l . \operatorname{inj} . \operatorname{dim}_{R} P \leqq$ $l$. inj. $\operatorname{dim}_{R} R / N^{2}<\infty$ for every projective $\left(R / N^{2}\right)$-module $P$ and that $l$. inj. $\operatorname{dim}_{R} N^{i-1} * N^{i}<\infty$ for $i \geqq 3$. Then $l F I D(R)=$ $l$. inj. $\operatorname{dim}_{R} R<\infty$. The method of proof uses a result of Eilenberg and a result of Bass on direct limits of modules together with the lemma: If $M$ is a left $R$-module such that $N^{k-1} M \neq 0$ and $N^{k} M=0$, then every simple direct summand of $N^{k-1}$ is isomorphic to a direct summand of $N^{k-1} / N^{k}$.

1. We begin by discussing some further properties of perfect and

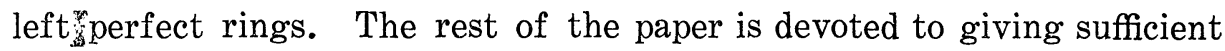
conditions for finiteness and equality of certain finitistic global dimensions for a semi-primary ring.

Let $R$ be a ring (with identity). By an $R$-module we shall always mean a left unitary module over $R$. In ([7]) and ([10]), Eilenberg and Nakayama define what they called minimal epimorphisms. Bass ([1]) altered this definition to call minimal epimorphisms projective covers. Eilenberg ([7)] studied the dimension theory for modules having minimal epimorphisms and said that a category of modules is perfect if every member of the category has a projective cover. Thus Bass ([1]) called a ring $R$ for which every $R$-module has a projective cover a left perfect ring. There are two special types of left perfect rings about which we are particularly interested. One is the semi-primary ring $R$ where the Jacobson radical ( $J$-radical) $N$ is nilpotent and $R / N$ is semi-simple with minimum condition (semi-simple), and the other is a ring with minimum condition on left ideals (left Artinian ring).

We define the following finitistic global dimensions for $R$, using the definitions and notation of ([1]) and ([3]). $l F P D(R)=\sup l . \operatorname{dim}_{R} M$ for all $R$-modules of finite projective dimension, $l f P D(R)=\sup l . \operatorname{dim}_{R} M$ for all finitely generated (f.g.) $R$-modules of finite projective dimension, $l F W D(R)=\sup w . l . \operatorname{dim}_{R} M$ for all $R$-modules of finite weak dimension, $l F I D(R)=\sup l$. inj. $\operatorname{dim}_{R} M$ for all $R$-modules of finite injective dimension.

In $\S 2$ we discuss some further properties of left perfect and perfect rings.

Received January 17, 1964. 
In $\S 3$ we give a partial answer to the following questions of Rosenberg and Zelinsky.

(1) Does lfPD $(R)=l F P D(R)$ ?

(2) Is $l f P D(R)$ finite?

We prove that if $R$ is a semi-primary ring with $J$-radical $N$ such that $N^{i} / N^{i+1}$ has finite projective dimension for $i \geqq 2$, then $m \leqq l f P D(R)=$ $l F P D(R) \leqq(m+1)$ where $m=\sup \left\{l_{\circ} \operatorname{dim}_{R} C: C\right.$ is a simple $R$-module of finite projective dimension\}.

In $\S 4$ we prove in a manner similar to $\S 3$ that if $R$ is a left Artinian ring with $J$-radical $N$ such that $N^{i} / N^{i+1}$ has finite injective dimension for $i \geqq 2$ and $R$ has finite self-injective dimension, then $l F I D(R)=l . \operatorname{inj} . \operatorname{dim}_{R} R$. We also show that if $R$ is a ring such that the direct product of projectives is projective, if the $J$-radical $N$ of $R$ has the property that $N^{i} / N^{i+1}$ has finite injective dimension for $i \geqq 2$, and if $R$ has finite self-injective dimension, then $l F I D(R)=l$. inj. $\operatorname{dim}_{R} R$. We conclude by giving examples for the above theorems.

This paper is essentially the first half of the author's dissertation at the University of Washington, Seattle, and was written during his tenure as a National Science Foundation Cooperative Graduate Fellow. The author wishes to express his gratitude to Professor James P. Jans for his advice and encouragement during the preparation of the dissertation.

2. Left perfect rings. Eilenberg ([7]) and Bass ([1]) introduced the following concepts.

Definition 2.1. A submodule $B$ of an $R$-module $A$ is called superfluous if $B+C=A$ implies $C=A$ whenever $C$ is a submodule of $A$. An $R$-homomorphism $f: A \rightarrow D$ is called minimal if $\operatorname{Ker} f$ is superfluous in $A$. If $A$ is projective and $f$ is an epimorphism, then $f$ is called a minimal epimorphism. The ring $R$ is called left perfect if every $R$-module has a minimal epimorphism.

Definition 2.2. An ideal $N$ of a ring $R$ is called left (right) $T$-nilpotent if given any sequence $\left\{a_{i}\right\}$ of elements in $N$, we can find an $n$ such that $a_{1} a_{2} \cdots a_{n}=0\left(a_{n} \cdots a_{2} a_{1}=0\right)$.

Bass proved the following theorem.

Theorem 2.3 ([1, Theorem P, p. 467]): Let $R$ be a ring and $N$ its J-radical. Then the following are equivalent.

(1) $N$ is left $T$-nilpotent and $R / N$ is semi-simple.

(2) $R$ is left perfect. 
(3) Every $R$-module has the same weak as projective dimension.

(4) A direct limit of $R$-modules of projective dimension $\leqq n$ has projective dimension $\leqq n$.

(5) $R$ has no infinite sets of orthogonal idempotents, and every nonzero right $R$-module has nonzero socle (sum of all simple submodules of the right $R$-module).

In [7] Eilenberg generalized the concept of semi-primary ring, and generalized a number of theorems of M. Auslander. We state two of them here in a slightly more restricted situation.

Proposition 2.4 ([7, Theorem 11, p. 333]). Let $R$ be a left perfect ring with $J$-radical $N$. If $A$ is an $R$-module, then the following are equivalent.

(1) $\operatorname{Ext}_{R}^{n+1}(A, R / N)=0$ where $R / N$ is considered as an $R$-module.

(2) $\operatorname{Tor}_{n+1}^{R}(R / N, A)=0$ where we consider $R / N$ as a right $\mathrm{R}$-module.

(3) $l \cdot \operatorname{dim}_{R} A \leqq n$.

Proposition 2.5 ([7, Theorem 12, p. 334]): Let $R$ be a perfect (i.e., left and right perfect) ring with $J$-radical $N$. Then the following are equivalent.

(1) l.gl。dim $R \leqq n$.

(2) $l . \operatorname{dim}_{R} C=w . l . \operatorname{dim}_{R} C \leqq n$ for all simple $R$-modules $C$.

(3) $l \cdot \operatorname{dim}_{R}(R / N)=w \cdot l .(R / N) \leqq n$ where we consider $R / N$ as an $R$-module.

(4) $l . \operatorname{dim}_{R} N=w . l \cdot \operatorname{dim}_{R} N \leqq n$.

(5) l.inj. $\operatorname{dim}_{R}(R / N) \leqq n$ where $R / N$ is considered as an $R$-module.

(6) $r \cdot \operatorname{dim}_{R}(R / N)=w \cdot r \cdot \operatorname{dim}_{R}(R / N) \leqq n$ where we treat $R / N$ as a right $R$-module.

REMARKs. From Proposition 2.4 it is clear that for a left perfect ring $R, l . g l \cdot \operatorname{dim} R \leqq w . r \cdot \operatorname{dim}_{R}(R / N) \leqq r \cdot \operatorname{dim}_{R}(R / N) \leqq r . g l \cdot \operatorname{dim} R$. By interchanging the $l$ and the $r$ in Proposition 2.5, we see that l. gl. $\operatorname{dim} R=r$. gl. $\operatorname{dim} R=$ gl. $\operatorname{dim} R$ for a perfect $\operatorname{ring} R$.

The following proposition asserts that the simple modules of a right perfect ring serve as test modules for determining injective dimensions of modules.

Proposition 2.6. Let $R$ be a right perfect ring with $J$-radical $N$. If $A$ is an $R$-module, then the following statements are equivalent.

(a) $\operatorname{Ext}_{R}^{n+1}(C, A)=0$ for all simple $R$-modules $C$.

(b) $\operatorname{Ext}_{R}^{n+1}(R / N, A)=0$.

(c) $l$. inj. $\operatorname{dim}_{R} A \leqq n$.

Furthermore if $n \geqq 1$, then (b) becomes $\operatorname{Ext}_{R}^{n+1}(R / N, A) \cong \operatorname{Ext}_{R}^{n}(N, A)=0$. 
Proof. (a) $\Leftrightarrow$ (b) and (c) $\Rightarrow$ (a) are obvious. We shall show that (a) $\Rightarrow(\mathrm{c})$

It is well known ([6]) that we can embed any $R$-module in an injective $R$-module. Thus it is possible to form the exact $\mathrm{R}$-sequence:

$$
0 \rightarrow A \stackrel{d_{0}}{\longrightarrow} Q_{0} \stackrel{d_{1}}{\longrightarrow} Q_{1} \stackrel{d_{2}}{\longrightarrow} \cdots \stackrel{d_{n-1}}{\longrightarrow} Q_{n-1} \stackrel{d_{n}}{\longrightarrow} Q_{n} \longrightarrow 0
$$

where $Q_{i}, 0 \leqq i \leqq(n-1)$, are injective. $\operatorname{Ext}_{R}^{n+1}(M, A) \cong \operatorname{Ext}_{R}^{1}\left(M, Q_{n}\right)$ for all $R$-modules $M$ where we use the exact sequences

$$
0 \longrightarrow A \longrightarrow Q_{0} \longrightarrow \operatorname{Im} d_{1} \longrightarrow 0
$$

and

$$
0 \longrightarrow \operatorname{Im} d_{i} \longrightarrow Q_{i} \longrightarrow \operatorname{Im} d_{i+1} \longrightarrow 0, \quad 1 \leqq i \leqq(n-1) .
$$

If we can show that $Q_{n}$ is injective, then $A$ would have injective dimension $\leqq n$.

It is well known ([3, Chapter I, Theorem 3.2, p. 8]) that $Q_{n}$ is injective if and only if for each left ideal $L$, each $R$-diagram

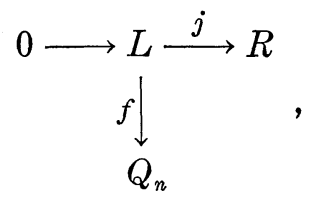

with $j$ the embedding map can be embedded in a commutative diagram

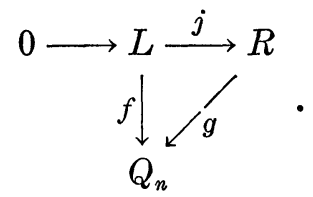

By using Zorn's Lemma (as in the proof of Theorem 3.2 in [3]), we can show that there exists a left ideal $L_{0}$ of $A$ containing $L$ such that

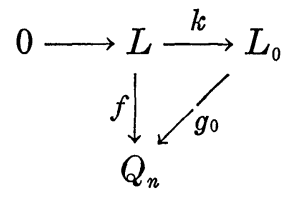

( $k$ the embedding map) is commutative and that $f$ cannot be extended to any left ideal of $R$ properly containing $L_{0}$. If $L_{0}=R$, then we are done. If $L_{0} \neq R$, then $R / L_{0} \neq 0$. According to Theorem 2.3, $R / L_{0}$ has nonzero socle $S\left(R / L_{0}\right)=S$.

$S=\bigoplus \Sigma C_{i}$ is the direct sum of simple $R$-modules. It is well-known 
that $\operatorname{Ext}_{R}^{1}\left(\bigoplus \Sigma C_{i}, Q_{n}\right) \cong \Pi \operatorname{Ext}_{R}^{1}\left(C_{i}, Q_{n}\right)$, which is the zero module. There exists a left ideal $L_{1}$ of $R$ containing $L_{0}$ such that $L_{1} / L_{0} \cong S$. $\operatorname{Ext}_{R}^{1}\left(S, Q_{n}\right)=0$ implies that we can extend $f$ (and $g_{0}$ ) to $L_{1}$, contradicting the maximality of $L_{0} . Q_{n}$ is therefore injective.

COROLlary 2.7. If $R$ is a right perfect ring with J-radical $N$, then l.gl. $\operatorname{dim} R=l . \operatorname{dim}_{R} R / N \geqq r . g l . \operatorname{dim} R$.

Proof. Since $l . g l . \operatorname{dim} R$ is the supremum of injective dimensions of all the $R$-modules and since $l . g l . \operatorname{dim} R \geqq l$. $\operatorname{dim}_{R} R$, it follows from Proposition 2.6 that $l . \mathrm{gl} . \operatorname{dim} R=l . \operatorname{dim}_{R} R$. The second part is essentially contained in a theorem proved by Eilenberg ([7, Theorem 12, p. 334]).

In [4] Chase proved some necessary and sufficient conditions that direct products of projective modules be projective. A module $A$ of a ring $R$ is called finitely related if there exists an exact sequence $0 \rightarrow$ $K \rightarrow F \rightarrow A \rightarrow 0$ of $R$-modules where $F$ is free and both $F$ and $K$ are $f . g$.

Proposition 2.8 ([4, Theorem 3.3, p. 467]). For any ring $R$ the following statements are equivalent.

(1) The direct product of any family of projective $R$-modules is projective.

(2) $R$ is left perfect and finitely generated right ideals of $R$ are finitely related.

Let $R$ be a ring satisfying (1) and (2) in Proposition 2.8. Let $\bigoplus \Sigma R_{a}(a \in X)$ be a direct sum of copies of $R$ as an $R$-module. Considering the exact sequence

$$
0 \longrightarrow \bigoplus \Sigma R_{a} \longrightarrow \Pi R_{a} \longrightarrow\left(\Pi R_{a}\right) /\left(\oplus \Sigma R_{a}\right) \longrightarrow 0,
$$

we note that $\left(\Pi R_{a}\right) /\left(\bigoplus \Sigma R_{a}\right)$ is the direct limit of projective $R$-modules and is therefore projective. The sequence splits, and $\bigoplus \Sigma R_{a}$ is embedded as a direct summand of $\Pi R_{a}$.

Proposition 2.9: Let $R$ be a ring satisfying (1) and (2) of Proposition 2.8. If $P$ is projective, then $l$. inj. $\operatorname{dim}_{R} P \leqq l$. inj. $\operatorname{dim}_{R} R$.

Proof. If $P$ is projective, then $P$ is a direct summand of a direct product $\Pi R_{a}$ copies of $R$. It then follows by an exercise in $C$ and $E$ ([3, Chapter VI, Exercise 7, p. 123]) that l. inj. $\operatorname{dim}_{R} R$.

CoRollary 2.10. Let $R$ be a ring as in Proposition 2.8. If $l F I D(R)$ and $l$. inj. $\operatorname{dim}_{R} R$ are both finite, then they are equal.

Proof. $\quad l$. inj. $\operatorname{dim}_{R} P \leqq l . \operatorname{inj} . \operatorname{dim}_{R} R \leqq l F I D(R)=n$ where $P$ is any 
projective $R$-module. Let $A$ be an $R$-module such that $l$.inj. $\operatorname{dim}_{R} A=$ $n$. Considering an exact sequence $0 \rightarrow K \rightarrow P \rightarrow A \rightarrow 0$ where $P$ is projective, we note that $l$. inj. $\operatorname{dim}_{R} K \leqq n$. Thus we get part of the exact sequence in Ext as follows:

$$
\operatorname{Ext}_{R}^{n}(B, K) \longrightarrow \operatorname{Ext}_{R}^{n}(B, P) \longrightarrow \operatorname{Ext}_{R}^{n}(B, A) \longrightarrow 0,
$$

where $B$ is an arbitrary $R$-module. But then $\operatorname{Ext}_{R}^{n}(B, P) \neq 0$ for an $R$-module $B$ such that $\operatorname{Ext}_{R}^{n}(B, A) \neq 0$. Hence $n \leqq l$. inj. $\operatorname{dim}_{R} P \leqq$ l. inj. $\operatorname{dim}_{R} R \leqq n$.

CoRollary 2.11. Let $R$ be as in Proposition 2.8. If $l F I D(R)=$ l. inj. $\operatorname{dim}_{R} R=0$, then $R$ is left Noetherian, i.e., $R$ is quasi-Frobenius ([9, Theorem 18, p. 11]).

Proof. According to theorem of Bass ([2, Theorem 1.1, p. 19]) $R$ is left Noetherian if and only if the direct sum of injective $R$-modules is injective. Let $\left\{Q_{i}: i \in I\right\}$ be a collection of injective $R$-modules. For each $i \in I$, we consider an exact sequence $0 \rightarrow K_{i} \rightarrow P_{i} \rightarrow Q_{i} \rightarrow 0$ where $P_{i}$ is projective and thus injective. Since $l_{0}$ inj. $\operatorname{dim}_{R} K_{i}$ is finite, $K_{i}$ is injective, and the sequence splits. Thus $Q_{i}$ is also projective, and $\bigoplus \Sigma Q_{i}(i \in I)$ is a projective $R$-module and hence an injective $R$-module.

3. Sufficient conditions that $l f P D(R)=l F P D(R)<\infty$. In this section we attempt to give relatively simple sufficient homological conditions to answer questions (1) and (2) of Rosenberg and Zelinsky (appearing in the introduction) in the affirmative. We have the following theorem.

THEOREM 3.1. Let $R$ be a semi-primary ring with J-radical $N$. If $l . \operatorname{dim}_{R}\left(N^{r-1} / N^{r}\right)<\infty$ for $r \geqq 3$, then $m \leqq l f P D(R)=l F P D(R) \leqq$ $(m+1)$ where $m=\max \left\{l \cdot \operatorname{dim}_{R} C: C\right.$ is a simple $R$-module of finite projective dimension\}.

Before we begin the proof of 3.1 we need a preliminary lemma.

Lemma 3.2. Let $R$ be a semi-primary ring with J-radical $N$ such that $N \neq 0$. If $M$ is an $R$-module such that $N^{r-1} M \neq 0$ and $N^{r} M=0$, then $N^{r-1} M$ is the direct sum of simple $R$-modules which appear as direct summands of $N^{r-1} / N^{r}$. Thus $N^{r-s} M / N^{r-s+1} M, r \geqq$ $(s-1)$, is the direct sum of simple $R$-modules which appear as direct summands of $N^{r-s} / N^{r-s+1}$.

Proof. The second part follows easily from the first part by noting that $N^{r-s+1}\left(M / N^{r-s+1} M\right)=0$ and $N^{r-s}\left(M / N^{r-s+1} M\right) \neq 0$. 
We first observe that $\left(N^{r-1} / N^{r}\right) M=\bigoplus \Sigma C_{i}(i \in I)$ is the direct sum of simple $R$-modules $C_{i}$. Let $x \in M$. Then the map $f_{x}: a \rightarrow a x, a \in N^{r-1} / N^{r}$, is an $R$-homomorphism of $N^{r-1} / N^{r}$ onto $\left(N^{r-1} / N^{r}\right) x$. Let $C_{i}, i \in I$, be one of the direct summands of $\left(N^{r-1} / N^{r}\right) M$. If $x_{0} \in C_{i}$, then $x_{0}=\Sigma a_{j} x_{j}(1 \leqq$ $j \leqq n$ ) where $a_{j} \in N^{r-1} / N^{r}$ and $x_{j} \in M$. Furthermore $x_{0}$ generates $C_{i}$.

Let $\varphi: L=\bigoplus \Sigma\left(N^{r-1} / N^{r}\right) \quad(n$ copies $) \rightarrow T=\left(N^{r-1} / N^{r}\right) x_{j}(1 \leqq j \leqq n)$ be the $R$-epimorphism given by $\varphi\left(\Sigma a_{j}(1 \leqq j \leqq n)\right)=\Sigma a_{j} x_{j}(1 \leqq j \leqq n)$. Since $L$ and $T$ are both modules over the semi-simple ring $R / N, L \cong$ $\operatorname{Ker} \varphi \oplus T . \quad C_{i}$ is a direct summand of $L$. By a well-known theorem ([10, Chapter IV, Theorem 2, p. 64]) $C_{i}$ is isomorphic to a direct summand of $N^{r-1} / N^{r}$.

Proof of Theorem 3.1. Let $M$ be an $R$-module of finite projective dimension. If $N M=0$, then $M$ is a direct sum of simple $R$-modules of finite projective dimension. Thus $M$ has projective dimension $\leqq m$ ([3, Chapter VI, Exercise 7, p. 123)].

Suppose then that $N M \neq 0$. We assert that $l$. $\operatorname{dim}_{R} N^{i} M \leqq m$ for $i \geqq 2$. If $N^{2} M=0$, then there is nothing to prove. Hence assume $N^{2} M \neq 0$. Let $t$ be the integer such that $N^{t} M=0$ and $N^{t-1} M \neq 0$. We have the submodules $N^{t-j} M, 1 \leqq j \leqq(t-2)$ to examine. We induce on the integer $j$. If $j=1$, the $N^{t-1} M$ is the direct sum of simple $R$-module of projective dimension $\leqq m$ by Lemma 3.2 and the hypotheses of the theorem. $l \cdot \operatorname{dim}_{R}\left(N^{t-1} M\right) \leqq m$, as above. Assume that l. $\operatorname{dim}_{R}\left(N^{t-j+1} M\right) \leqq m$ where $1<j \leqq(t-2) . \quad\left(N^{t-j} M\right) /\left(N^{t-j+1} M\right)$ has finite projective dimension $\leqq m$, being the direct sum of simple $R$-modules of projective dimension $\leqq m$ (Lemma 3.2 and the hypotheses of the theorem). From the exact sequence in Ext in the first variable for the exact sequence $0 \rightarrow N^{t-j+1} M \rightarrow N^{t-j} M \rightarrow\left(N^{t-j} M\right) /\left(N^{t-j+1} M\right) \rightarrow 0$ we see that $l . \operatorname{dim}_{R}\left(N^{t-j} M\right) \leqq m$. We have therefore proved our assertion. Since $l . \operatorname{dim}_{R}\left(N^{2} M\right) \leqq m$, from the exact sequence $0 \rightarrow N^{2} M \rightarrow M \rightarrow$ $M / N^{2} M \rightarrow 0$ we conclude that $l . \operatorname{dim}_{R} M / N^{2} M<\infty$.

From the exact sequence $0 \rightarrow N^{2} \rightarrow R \rightarrow R / N^{2} \rightarrow 0$ we notice in particular that $l . \operatorname{dim}_{R} N^{2} \leqq m$ and that $l \cdot \operatorname{dim}_{R}\left(R / N^{2}\right) \leqq m+1$. Now, $R / N^{2}$ as a ring is semi-primary with $J$-radical $N / N^{2}$, and $M / N^{2} M$ is an $R / N^{2}$-module. Thus $M / N^{2} M$ has a minimal epimorphism as an $R / N^{2}$ module. Let $0 \rightarrow K \rightarrow P \rightarrow M / N^{2} M \rightarrow 0$ be the minimal $\left(R / N^{2}\right)$-epimorphism for $M / N^{2} M$. Then $l . \operatorname{dim}_{R} P \leqq m+1$ (since $P$ is a direct summand of a direct sum of copies of $R / N^{2}$ as an $R$-module), and $K \subseteq\left(N / N^{2}\right) P$ ([7, p. 330]). $K=\bigoplus \Sigma C_{a}(a \in I)$ is a direct sum of simple $R$-modules $C_{a}$, and $l . \operatorname{dim}_{R} K<\infty$. Again applying an exercise in $C$ and $E$ ([3, Chapter VI, Exercise 7, p. 123]), we see that $l . \operatorname{dim}_{R} C_{a}<\infty$ for all $a \in I$. Therefore $l \cdot \operatorname{dim}_{R} K \leqq m$. Using the exact sequence in Ext for the exact sequences $0 \rightarrow K \rightarrow P \rightarrow M / N^{2} M \rightarrow 0$ and $0 \rightarrow N^{2} M \rightarrow M \rightarrow$ $M / N^{2} M \rightarrow 0$,əM conclude that $l . \operatorname{dim}_{R} M \leqq l \cdot \operatorname{dim}_{R} M / N^{2} M \leqq(m+1)$. 
Lastly we assert that $M / N^{2} M$ is the direct limit of $f . g . R$-modules of finite projective dimension. Let $P=\bigoplus \Sigma P_{\alpha}(\alpha \in \Gamma)$ be a direct decomposition of $P$ into $f . g$. projective $\left(R / N^{2}\right)$-modules (each of projective dimension $\leqq m+1)$. This fact follows from a result of Eilenberg ([7, Proposition 3, p. 330]). If $\Delta$ is an arbitrary finite subset of $\Gamma$ and if $J$ is any finite subset of $I$ such that $\bigoplus \Sigma C_{a}(a \in J) \subseteq \bigoplus \Sigma P_{\alpha}(\alpha \in \Delta)$, then $M / N^{2} M$ is the direct limit of $f . g . R$-modules $S(\Delta, J)$ where $0 \rightarrow$ $\bigoplus \Sigma C_{a}(a \in J) \rightarrow \bigoplus \Sigma P_{\alpha}(\alpha \in \Delta) \rightarrow S(\Delta, J) \rightarrow 0$ is exact. Since the first two both have finite projective dimension, so does $S(\Delta, J)$. From Theorem 2.3 it follows that $l . \operatorname{dim}_{R} M \leqq l . \operatorname{dim}_{R}\left(M / N^{2} M\right) \leqq l f P D(R)$. Since $M$ was arbitrary with finite projective dimension, we can write that $m \leqq$ $l f P D(R) \leqq l F P D(R) \leqq(m+1)$.

4. Sufficient conditions that $l F I D(R)<\infty$. We state the main theorem of this section.

THEOREM 4.1. Let $R$ be a semi-primary ring with radical $N$ such that

(a) for any projective $\left(R / N^{2}\right)$-module $P$,

$$
\text { l. inj. } \operatorname{dim}_{R} P \leqq l \text {. inj. } \operatorname{dim}_{R} R / N^{2}<\infty .
$$

(b) if $r \geqq 3$, then l. inj. $\operatorname{dim}_{R}\left(N^{r-1} / N^{r}\right)<\infty$.

Then $l$. inj. $\operatorname{dim}_{R} R<\infty$, and $l F I D(R)=l$. inj. $\operatorname{dim}_{R} R$.

Proof. Let $M$ be an $R$-module of finite injective dimension over $R$. Then it follows, in a manner similar to the proof of Theorem 3.1, that $l$. inj. $\operatorname{dim}_{R} N^{2} M \leqq m$ where $m=\max \left\{l\right.$. inj. $\operatorname{dim}_{R} C: C$ is a simple $R$-module of finite injective dimension\}. From the exact sequence $0 \rightarrow N^{2} \rightarrow R \rightarrow R / N^{2} \rightarrow 0$ it is evident that $l$.inj. $\operatorname{dim}_{R} N^{2} \leqq m$ and l. inj. $\operatorname{dim}_{R} R \leqq \max (m, n)<\infty$.

Obviously, l.inj. $\operatorname{dim}_{R}\left(M / N^{2} M\right)<\infty . M / N^{2} M$ is an $R / N^{2}$-module and therefore has a minimal $R / N^{2}$-epimorphism $0 \rightarrow K \rightarrow P \rightarrow M / N^{2} \rightarrow 0$. As in the proof of Theorem 3.2, $K$ is the direct sum of simple $R$ modules of finite injective dimension. $K$ is thus a direct summand of a direct product of simple $R$-modules of finite injective dimension, and we have that $l$. inj. $\operatorname{dim}_{R} K \leqq m$ ([3, Chapter VI, Exercise 7, p. 123]). From the exact sequence in Ext for the second variable applied to the exact sequence $0 \rightarrow K \rightarrow P \rightarrow M / N^{2} M \rightarrow 0$ we deduce that $l$.inj. $\operatorname{dim}_{R}\left(M / N^{2} M\right) \leqq$ $\max (m, n)$ and hence $l$. inj. $\operatorname{dim}_{R} M \leqq \max (m, n)$.

$$
l F I D(R) \leqq \max (m, n)<\infty,
$$

and by Corollary 2.10, lFID $(R)=l . \operatorname{inj} . \operatorname{dim}_{R} R$. 
We remark that a semi-primary ring $R$ satisfies condition (a) of Theorem 4.1 if $R$ is a left Artinian ring with $l$.inj. $\operatorname{dim}_{R}\left(R / N^{2}\right)=$ $n<\infty$, or if $R / N^{2}$ satisfies (1) and (2) of Proposition 2.8 and l. inj. $\operatorname{dim}_{R} R / N^{2}=n<\infty$. In the first case we apply the well-known fact $([2$, Theorem 1.1, p. 19]) that the direct sum of modules of injective dimension $\leqq n$ has injective dimension $\leqq n$ for Noetherian rings. Thus $P$ a direct summand of a free $R$-module implies that the injective dimension of $P$ is less than or equal to the injective dimension of $R$. In the second case we use the remarks prior to Proposition 2.9 together with an exercise in $\mathrm{C}$ and $\mathrm{E}$ ([3, Chapter VI, Exercise 7, p. 123]) to find that free $R$-modules and therefore projective $R$-modules have injective dimension less than or equal to the injective dimension of $R$.

5. Examples. In this section we give examples which satisfy Theorems 3.1 and 4.1 respectively. The construction is essentially that given by Chase in [5].

Let $R^{\prime}$ and $R^{\prime \prime}$ be rings and $X$ a left $R^{\prime}$-, right $R^{\prime \prime}$-bimodule. We form the ring $R$ consisting of matrices $\left(a^{\prime}, x, 0, z^{\prime \prime}\right)$ where $a^{\prime} \in R^{\prime}$, $x \in X$, and $a^{\prime \prime} \in R^{\prime \prime}$. Addition is componentwise and multiplication is given by the equation

$$
\left(a_{1}^{\prime}, x_{1}, 0, a_{1}^{\prime \prime}\right)\left(a_{2}^{\prime}, x_{2}, 0, a_{2}^{\prime \prime}\right)=\left(a_{1}^{\prime} a_{2}^{\prime}, a_{1}^{\prime} x_{2}+x_{1} a_{2}^{\prime \prime}, 0, a_{1}^{\prime \prime} a_{2}^{\prime \prime}\right) .
$$

Chase proved the following proposition.

Proposition 3.7 ([5, Lemma 4.1, p. 17]). Let $R$ be as above, and suppose further that $R^{\prime}$ is semi-primary (respectively Artinian) with radical $N^{\prime}$ and $R^{\prime \prime}$ is semi-simple (with minimum conditions). Then $R$ is semi-primary (respectively Artinian) with radical $N$ consisting of the matrices $\left(a^{\prime}, x, 0,0\right)$ where $a^{\prime} \in N^{\prime}$ and $x \in X$. Moreover gl. $\operatorname{dim} R=$ $\max \left(\mathrm{gl} . \operatorname{dim} R^{\prime}, 1+l . \operatorname{dim}_{R} X\right)$.

If $G$ is the finite group of order 2 and $K$ is a field of characteristic 2 , then $K(G)$, the group algebra, is a quasi-Frobenius algebra with nonzero radical $N(G)$ such that $N(G)^{2}=0([9$, p. 7]). Eilenberg et al. ([8, Proposition 15, p. 94]) have shown that for each positive integer $m$, there exists a semi-primary ring $R_{m}^{\prime}$ with radical $N_{m}^{\prime}$ such that gl. $\operatorname{dim} R_{m}^{\prime}=m$ and $\left(N_{m}^{\prime}\right)^{2} \neq 0$. Let $R^{\prime}=K(G) \oplus R^{\prime \prime}$ (ring direct sum). The radical of $R^{\prime}$ is $N^{\prime}=N(G)+N_{m}^{\prime}$. We can suppose that $R_{m}^{\prime}$ is a finite dimensional algebra over $K$. Then $R^{\prime}$ is a vector space over $K$.

(i) Let $R^{\prime}=R^{\prime}, X=N^{\prime}$ and $R^{\prime \prime}=K$ in Proposition 3.7. Then the following facts hold.

(a) gl. $\operatorname{dim} R=l \cdot \operatorname{dim}_{R} N=\infty$.

(b) $l . \operatorname{dim}_{R} N^{j}=l . \operatorname{dim}_{R^{\prime}}\left(N^{\prime}\right)^{j} \leqq(m-1)$ if $j \geqq 2$.

(c) l. inj. $\operatorname{dim}_{R} R=\max \left\{l\right.$. inj. $\operatorname{dim}_{R^{\prime}} R^{\prime}, l$. inj. $\left.\operatorname{dim}_{R^{\prime}} N^{\prime}\right\}$

$=l$. inj. $\operatorname{dim}_{R^{\prime}} N^{\prime}=\infty$. 
Thus we have an example of a semi-primary (Artinian) ring satisfying the hypotheses of Theorem 3.1 but not those of Theorem 4.1.

(ii) Suppose that $\left(N^{\prime}\right)^{q}=\left(N_{m}^{\prime}\right)^{q} \neq 0$ and $\left(N^{\prime}\right)^{q+1}=\left(N_{m}^{\prime}\right)^{q+1}=0$ where $q \geqq 2$. Let $R^{\prime}=R^{\prime}, X=\left(N^{\prime}\right)^{q}$ and $R^{\prime \prime}=K$ in Proposition 3.7. Then the following facts hold.

(a) gl. $\operatorname{dim} R=l \cdot \operatorname{dim}_{R} N=\infty$.

(b) l. inj. $\operatorname{dim}_{R} R=l$. inj. $\cdot_{R^{\prime}} R^{\prime}=m$.

(c) $l$. inj. dim $N^{j}=l$. inj. $\operatorname{dim}_{R^{\prime}}\left(N^{\prime}\right)^{j} \leqq m$.

This gives an example of an Artinian ring satisfying the hypotheses of Theorem 4.1.

\section{REFERENCES}

1. H. Bass, Finitistic dimension and a homological generalization of semi-primary rings, Trans. Amer. Math. Soc. 90 (1960).

2. - Injective dimension in Noetherian rings, Trans. Amer. Math. Soc. 102 (1962), 18-29.

3. H. Cartan and S. Eilenberg, Homological algebra, Princeton University Press, Princeton, 1956.

4. S. Chase, Direct products of modules, Trans. Amer. Math. Soc. 97 (1960), 457-473.

5. - A generalization of the ring of triangular matrices, Nagoya Math. J. 18 (1961), 13-25.

6. B. Eckmann and A. Schopf, Über injective moduln, Arch. Math. 4 (1953), 75-78.

7. S. Eilenberg, Homological dimensions and syzygies, Ann. of Math. 64 (1956), 328-336.

8. S. Eilenberg, H. Nagao and T. Nakayama, On the dimension of modules and algebras, IV: Dimension of residue rings of hereditary rings, Nagoya Math. J. 10 (1956), 87-95.

9. S. Eilenberg and T. Nakayama, On the dimension of modules and algebras, II: Frobenius algebras and quasi-Frobenius algebras, Nagoya Math. J. 9 (1955), 1-16.

10. - On the dimension of modules and algebras, $V$ : Dimension of residue rings, Nagoya Math. J. 11 (1957) 9-12.

11. N. Jacobson, Structure of rings, Amer. Soc. Colloquium Publications, 37, Providence, 1956. 


\section{PACIFIC JOURNAL OF MATHEMATICS}

\section{EDITORS}

H. Samelson

Stanford University

Stanford, California

R. M. Blumenthal

University of Washington

Seattle, Washington 98105
J. Dugundu

University of Southern California Los Angeles, California 90007

*Richard Arens

University of California

Los Angeles, California 90024

\section{ASSOCIATE EDITORS}
E. F. BECKENBACH
B. H. NeumanN
F. WOLF
K. YOSIDA

\section{SUPPORTING INSTITUTIONS}

UNIVERSITY OF BRITISH COLUMBIA CALIFORNIA INSTITUTE OF TECHNOLOGY UNIVERSITY OF CALIFORNIA MONTANA STATE UNIVERSITY

UNIVERSITY OF NEVADA

NEW MEXICO STATE UNIVERSITY

OREGON STATE UNIVERSITY

UNIVERSITY OF OREGON

OSAKA UNIVERSITY

UNIVERSITY OF SOUTHERN CALIFORNIA
STANFORD UNIVERSITY

UNIVERSITY OF TOKYO

UNIVERSITY OF UTAH

WASHINGTON STATE UNIVERSITY

UNIVERSITY OF WASHINGTON

AMERICAN MATHEMATICAL SOCIETY CALIFORNIA RESEARCH CORPORATION SPACE TECHNOLOGY LABORATORIES NAVAL ORDNANCE TEST STATION 


\section{Pacific Journal of Mathematics}

\section{Vol. 15, No. $1 \quad$ September, 1965}

Donald Charles Benson, Unimodular solutions of infinite systems of linear

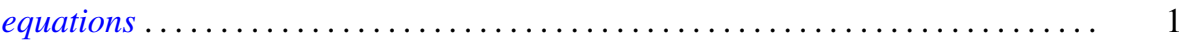

Richard Earl Block, Transitive groups of collineations on certain designs . . . . . . 13

Barry William Boehm, Existence of best rational Tchebycheff approximations .... . 19

Joseph Patrick Brannen, A note on Hausdorff's summation methods . . . . . . . . . . 29

Dennison Robert Brown, Topological semilattices on the two-cell ............ 35

Peter Southcott Bullen, Some inequalities for symmetric means . . . . . . . . . . 47

David Geoffrey Cantor, On arithmetic properties of coefficients of rational

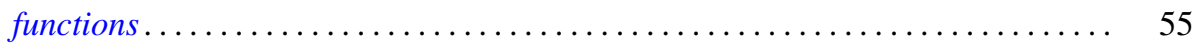

Luther Elic Claborn, Dedekind domains and rings of quotients . . . . . . . . . 59

Allan Clark, Homotopy commutativity and the Moore spectral sequence ........ 65

Allen Devinatz, The asymptotic nature of the solutions of certain linear systems of

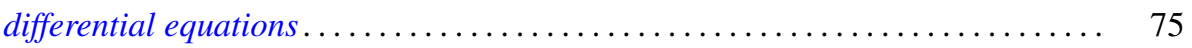

Robert E. Edwards, Approximation by convolutions ................... 85

Theodore William Gamelin, Decomposition theorems for Fredholm operators . . . . . 97

Edmond E. Granirer, On the invariant mean on topological semigroups and on

topological groups .................................. 107

Noel Justin Hicks, Closed vector fields . . . . . . . . . . . . . . . . . . . 141

Charles Ray Hobby and Ronald Pyke, Doubly stochastic operators obtained from

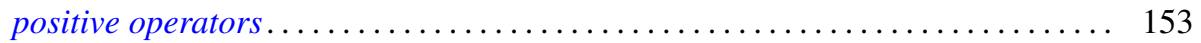

Robert Franklin Jolly, Concerning periodic subadditive functions . . . . . . . . . 159

Tosio Kato, Wave operators and unitary equivalence . . . . . . . . . . . . . . 171

Paul Katz and Ernst Gabor Straus, Infinite sums in algebraic structures . . . . . . . 181

Herbert Frederick Kreimer, Jr., On an extension of the Picard-Vessiot theory ...... 191

Radha Govinda Laha and Eugene Lukacs, On a linear form whose distribution is

identical with that of a monomial ......................... 207

Donald A. Ludwig, Singularities of superpositions of distributions . . . . . . . . . 215

Albert W. Marshall and Ingram Olkin, Norms and inequalities for condition

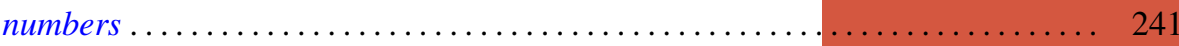

Horace Yomishi Mochizuki, Finitistic global dimension for rings . . . . . . . . . . 249

Robert Harvey Oehmke and Reuben Sandler, The collineation groups of division

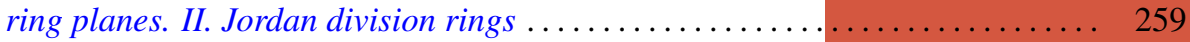

George H. Orland, On non-convex polyhedral surfaces in $E^{3} \ldots \ldots \ldots \ldots \ldots \ldots \ldots 267$

Theodore G. Ostrom, Collineation groups of semi-translation planes . . . . . . . . 273

Arthur Argyle Sagle, On anti-commutative algebras and general Lie triple

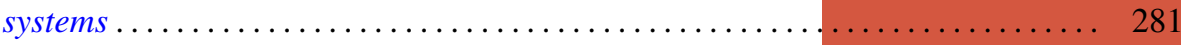

Laurent Siebenmann, A characterization of free projective planes . . . . . . . . . 293

Edward Silverman, Simple areas.................................. 299

James McLean Sloss, Chebyshev approximation to zero .................. 305

Robert S. Strichartz, Isometric isomorphisms of measure algebras . . . . . . . . . 315

Richard Joseph Turyn, Character sums and difference sets . . . . . . . . . . . . 319

L. E. Ward, Concerning Koch's theorem on the existence of arcs . . . . . . . . . . 347

Israel Zuckerman, A new measure of a partial differential field extension ......... 357 